Classification

Physics Abstracts

$61.16-82.80$

\title{
Medium-voltage field-emission STEM - the ultimate AEM
}

\author{
H.S. von Harrach \\ VG Microscopes, Fisons Instruments, East Grinstead RH19 1UB, U.K.
}

(Received March 29; accepted April 25, 1994)

\begin{abstract}
V}$ cold field-emission STEMs have achieved microanalysis of materials at the nanometre level in recent years. $300 \mathrm{kV}$ STEMs have now been developed to improve the analytical capabilities towards the ultimate goal of atomic sensitivity. The HB603 cold field-emission (CFE) STEMs were optimised for analytical microscopy and results at $300 \mathrm{kV}$ show an improvement in Xray peak-to-background ratio by a factor of 2, and an EELS resolution of $0.4 \mathrm{eV}$. In the $Z$-contrast imaging mode the high-resolution instrument (HB603U) achieves a point resolution of $0.13 \mathrm{~nm}$.
\end{abstract}

\section{Introduction.}

Field-emission STEMs have been used for many years to achieve the highest analytical performance obtainable by electron microscopy. The VG HB501 series of instruments was designed as $100 \mathrm{kV}$ cold field-emission STEMs to exploit the high source brightness, achieving high resolution $\mathrm{X}$-ray microanalysis at the nanometre level [1]. Similarly, for electron energy loss spectroscopy (EELS) the cold field-emission (CFE) source with an energy spread of a quarter of a volt provides higher energy resolution than any other source available [2]. Recently, the $Z$-contrast technique developed by Pennycook [3] using the HB501 has provided a new method for both microanalysis and high-resolution STEM imaging at the atomic level.

Medium voltage (300 - $400 \mathrm{kV})$ transmission electron microscopes were developed in the $1980 \mathrm{~s}$ with the aim of improving the image resolution and microanalysis of $100 \mathrm{kV}$ TEM/STEMs. However, these instruments failed to meet the improvements in analytical performance predicted by theory [4]. A CFE STEM, the VG HB603, was developed as a $300 \mathrm{kV}$ instrument for the highest analytical performance and, as a high-resolution version (HB603U), also for atomic resolution by $Z$-contrast imaging.

\section{Design of a $300 \mathrm{kV}$ CFE STEM.}

The VG HB603 series of STEMs is fully computer controlled including the gun and HT system, and has an advanced graphical user interface (GUI). The instruments are ion pumped in the gun and the column (diffusion pump option for the column). Ultra-high vacuum is achieved 


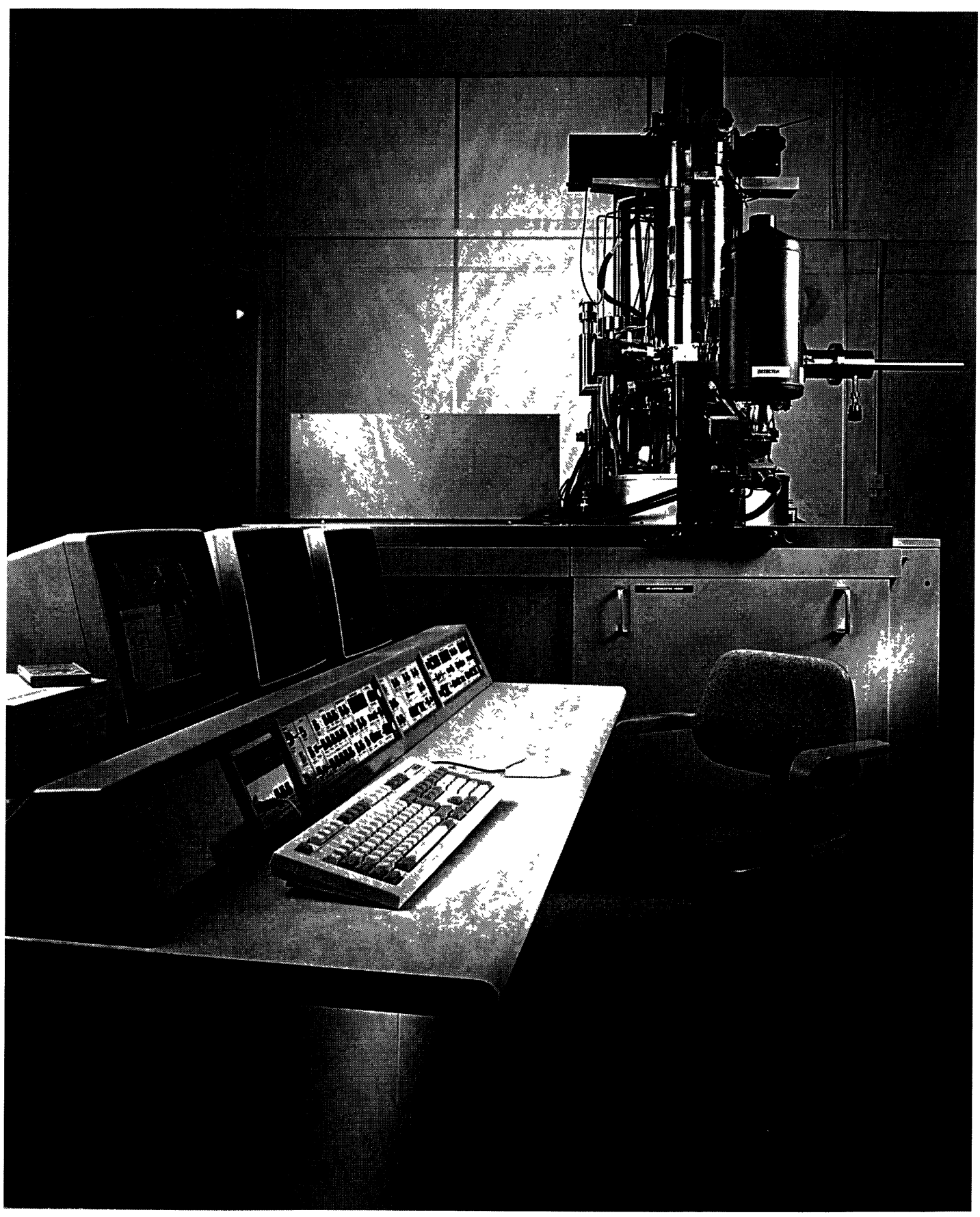

Fig. 1. - The HB603 CFE STEM. 
throughout the instrument to ensure the clean environment which is essential for meaningful high-resolution microanalysis. An automatic bake-out cycle makes achievement of UHV a simple routine.

Figure 1 shows the analytical instrument (HB603) and figure 2 is a schematic section of the column.

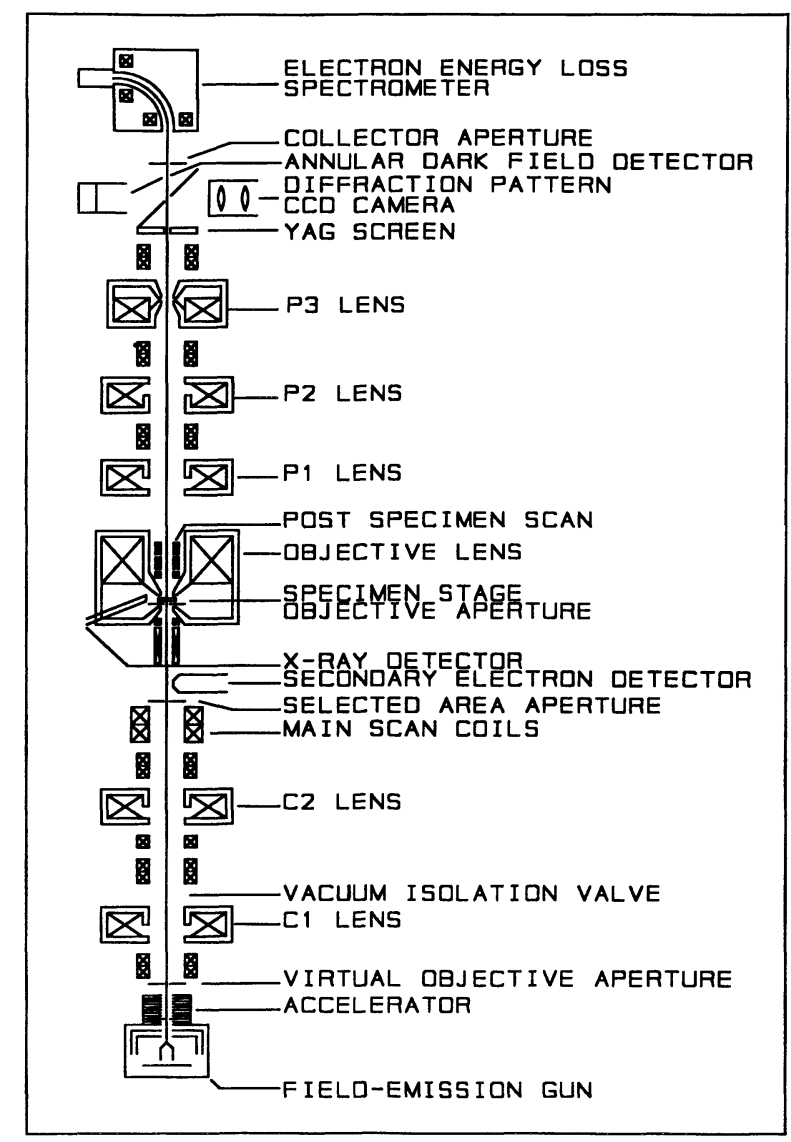

Fig. 2. - Schematic of HB603 column.

2.1 CFE GUN. - The cold field-emission gun is the key element in the design of this instrument. It uses standard UHV technology to achieve better performance than both Schottky and thermal field-emission guns in

a) brightness $>10^{9} \mathrm{amp} / \mathrm{cm}^{2} / \mathrm{sr}$ compared to $10^{8}$

b) source energy spread $0.25 \mathrm{eV}$ compared to about $0.8 \mathrm{eV}$;

c) source life-time 1 - 2 years with patented degassing technique

d) start-up time 5 - 10 seconds for automatic tip cleaning compared to 1.5 hours for Schottky FEG. 
The CFE gun produces an electron beam of typically 5-10 $\mu \mathrm{A}$ which is focused through a differential pumping aperture within an 11 stage accelerator stack. In order to pump the source effectively to pressures around $10^{-11} \mathrm{mb}$ a wide-bore high-voltage tube below the gun provides an efficient pumping path to the ion pump (see Fig. 2). The accelerator is pumped separately and provides further isolation from the column vacuum. Under these conditions the beam current noise is reduced to less than $2 \%$ and the long-term drift to about $5 \%$ per hour without applying current compensation.

2.2 Column. - The electron beam is focused on to the specimen by two condenser lenses and a condenser-objective. A range of preset probe sizes from 0.2 to $20 \mathrm{~nm}$ can be selected, but continuous control of all lenses at 16 bit precision ( 20 bit for the objective lens) is also available to optimise the probe for specific conditions.

In order to achieve the best performance for X-ray microanalysis the objective polepieces, specimen holder and X-ray detector have been designed for maximum sensitivity and peak-tobackground $(P / B)$ ratio. The result is a collection angle of 0.3 srad. - almost double that of any previous AEM - at a take-off angle of $20^{\circ}$. In addition, special precautions were taken to minimise the stray X-rays which could reach the detector, i.e. to minimise the "hole" count, by fitting light element materials (e.g. beryllium) in front of all apertures and around the specimen, the specimen holder and the polepieces. A crucial feature of the design is the use of a virtual objective aperture which defines the probe convergence just above the gun, i.e. a considerable distance from the specimen, such that few of the X-rays generated at the edge of the aperture can reach the specimen.

In the HB603 (analytical version) the space available for the side-entry stage allows for high tilt angles of the specimen: $\pm 60^{\circ}$ about the eucentric axis and $\pm 45^{\circ}$ about the second axis. The stage is a UHV side-entry goniometer with a detachable specimen holder which is loaded from an airlock or preparation chamber. The detachable holder design ensures isolation from the environment and reduces the specimen drift rate to extremely low values, $<0.2 \mathrm{~nm} / \mathrm{min}$, which is important for high-resolution microanalysis.

For the HB603U (high-resolution version) the objective lens has a spherical aberration coefficient of $C_{\mathrm{s}}=1.0 \mathrm{~mm}$ and the specimen tilt range of the eucentric double-tilt holder is reduced to $\pm 10^{\circ}$ on each axis.

The function of the STEM projector system, unlike that of a CTEM instrument, is simply to transfer the cone of transmitted electrons to the detectors, rather than forming an image on a screen. This means that the type of signal detected, i.e. bright- field or dark-field, can be selected by different geometries of annular detector. The projector lenses of the HB603 provide enormous flexibility in the range of camera lengths and detection angles. The instrument has three YAG scintillator detectors, a central small-angle detector for bright-field, a wide-angle annular darkfield detector and a screen for diffraction patterns. The angular range for diffraction patterns and the $\mathrm{ADF}$ detector can be varied from $\pm 10^{\circ}$ to $\pm 0.3^{\circ}$, corresponding to camera lengths of 50 to $2000 \mathrm{~mm}$. Diffraction patterns from areas of less than $0.5 \mathrm{~nm}$ can be recorded via a high-sensitivity CCD camera or photographic film.

2.3 CONTROL SYSTEM. - The control system is designed to provide maximum flexibility for the operator by a combination of direct front panel control and access via a graphical user interface. For controlling the functions to which the operator needs frequent access the ergonomic arrangement of the front panels (see Fig. 1) provides rapid access and ease of control. Preset conditions, such as probe sizes and camera lengths, can be selected instantly but in addition total control of all lens, alignment and stigmator settings is provided. For the less frequently used functions, such as calibration, a mouse-driven GUI allows the user to control parameters and handle data files. 
The control system, as shown schematically in figure 3 is based on two computers: one for instrument control, operating under OS/2, the second for control of the image acquisition and display. The two computers are linked by an Ethernet line which can also be connected to an external computer for down-loading images. The instrument controller communicates with the high-voltage generator, the column electronics and the stage stepper motors via optically isolated serial lines (RS232). The system is interrupt driven to minimise the communication traffic which has the potential for causing interference in high-stability systems.

High-voltage and source conditioning are implemented by automatic cycles in which potential error conditions and vacuum excursions are monitored.

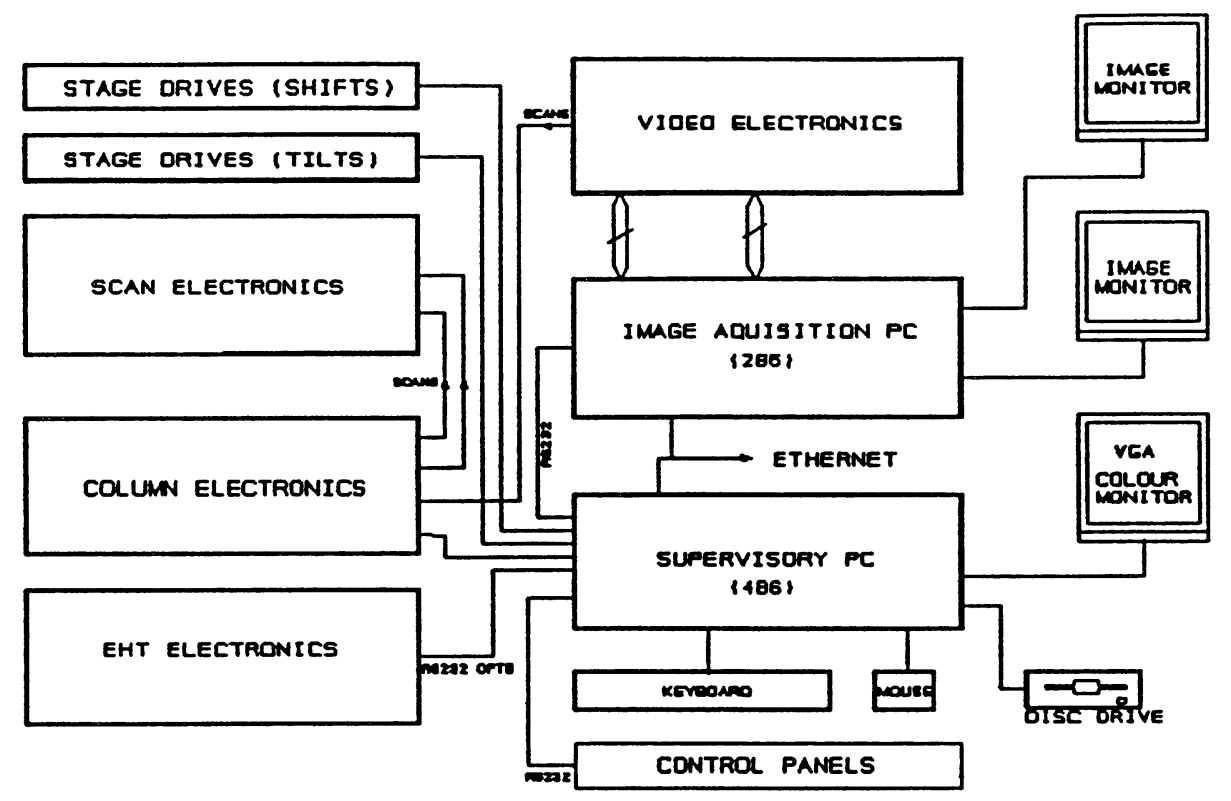

Fig. 3. - Schematic of the HB603 control system.

The imaging system contains two framestores for displaying two independent signals which can be acquired at up to nominally $2000 \times 2000$ pixels per frame and 12 bit resolution. The signals can be analogue or digital and may be normalised to a third signal, e.g. for beam current compensation. At TV rate recursive filtering can be used for noise reduction. Images are stored on the computer hard disc and can be transferred to a high-definition printer or an external computer.

\section{Analytical performance.}

3.1 X-RAY MICROANALYSIS. - The X-ray performance was tested with $\mathrm{Si}(\mathrm{Li})$ and Ge Oxford Instruments windowless detectors at 100 to $300 \mathrm{kV}$ using a standard chromium film on a copper grid. The peak-to-background $(P / B)$ ratio of the $\mathrm{Cr} \mathrm{K}_{\alpha}$ peak is a well-established test of the sensitivity attainable by an electron microscope. The $P / B$ ratio as defined by the Fiori method [5] increases from about 3500 at $100 \mathrm{kV}$ to 6000 at $300 \mathrm{kV}$, as shown in figure 4. As reported by Williams and Steel [4], thirteen other AEMs show no increase of $P / B$ ratio with voltage whereas 
the $\mathrm{HB} 603$ values almost double over the 100 to $300 \mathrm{kV}$ range. The combination of high $P / B$ ratio with large collection angles gives the $\mathrm{HB} 603$ a sensitivity for X-ray detection of at least three times higher than for any other AEM [6].

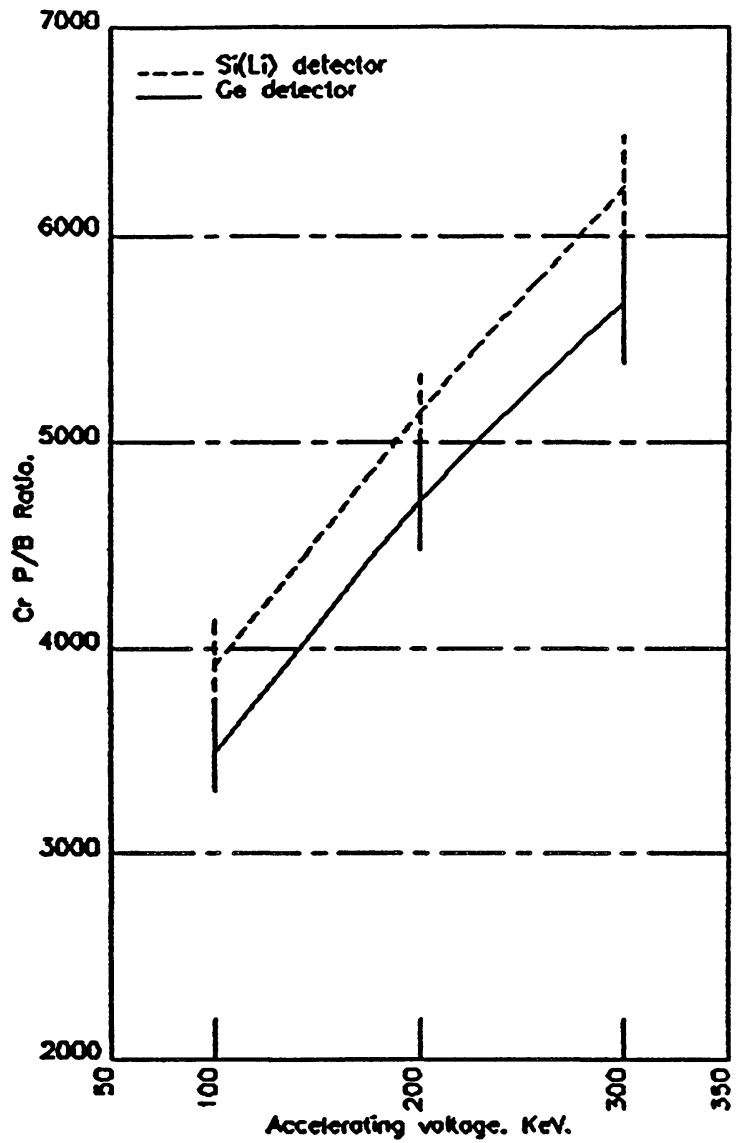

Fig. 4. $-P / B$ ratio as a function of acceleration voltage for $\mathrm{Si}(\mathrm{Li})$ and $\mathrm{Ge}$ detectors $(100 \mathrm{~nm} \mathrm{Cr}$ film $)$.

Another crucial test of X-ray performance is that of spurious counts, i.e. the number of X-rays detected when the beam passes through a thin film surrounded by a thick specimen. This is a more critical test than the usual "hole count" measurements which depend on the individual specimen geometry. Our measurements were carried out with a $30 \mathrm{~nm}$ chromium film deposited on a holey carbon film on a gold 400-mesh grid, backed by a thick gold ring with $1 \mathrm{~mm}$ diameter hole [7]. The beam was positioned at the centre of a grid square and the ratio of AuL counts in 100 seconds with the probe in a hole to $\mathrm{CrK}$ counts with the probe on the film was recorded in figure 5 at 100 to 300 $\mathrm{kV}$. The comparison with a TEM/STEM measurement under identical conditions [7] in figure 5 shows the fraction of spurious X-rays to be up to 10 times lower with the HB603.

The collection efficiency of the HB603 detector system is so high that count rates of 5-10 kcps are not unusual. At such high input count rates the X-ray analyzer normally has a dead time exceeding 50\%. VG Microscopes have developed the MaxiCount ${ }^{\mathrm{TM}}$ technique, in conjunction with Lehigh University [8], to improve the throughput by blanking the incident beam during the 


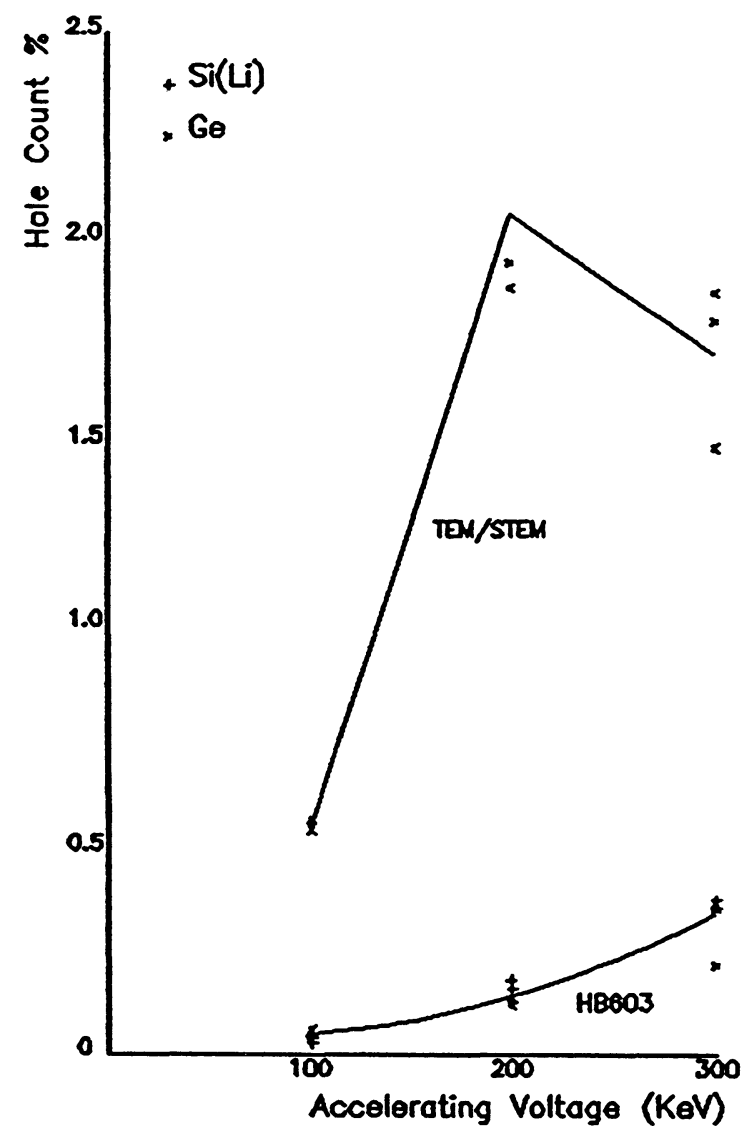

Fig. 5. - Ratio of AuL to CrK counts (\% hole count) for HB603 and typical TEM/STEM at 100 to $300 \mathrm{kV}$ (TEM/STEM data from Ref. [7]).

time interval that the pulse processor is busy. The result, shown in figure 6 , is a throughput within $10 \%$ of the theoretical maximum up to about $20 \mathrm{kcps}$ and a maximum output count rate of 30 $\mathrm{kcps}$, depending on processor time. This technique increases the detection efficiency by up to 3.5 times.

3.2 EELS PERFORMANCE. - EELS has become a powerful analytical technique in recent years and the CFE gun has contributed significantly in improving the energy resolution of energy-loss spectra due to its narrow source energy spread $(0.25 \mathrm{eV})$. The high brightness of the CFE gun has led to improvements in spatial resolution to sub-nanometre levels.

The energy resolution of the $\mathrm{HB} 603$ fitted with a second-order corrected magnetic sector spectrometer (VG ELS6030) and serial detector was measured at $300 \mathrm{kV}$. Figure 7 shows the low-loss region of an aluminium spectrum for a 1 second exposure; the zero-loss peak has a width of 0.4 eV FWHM and demonstrates the high-voltage stability of $1 \mathrm{ppm}$.

At $300 \mathrm{kV}$ the electron mean free path is roughly double that at $100 \mathrm{kV}$ [9] and consequently the single scattering regime extends to sample thicknesses of 50-100 nm. EELS data can therefore be analysed quantitatively for specimens which are much easier to prepare and are more representative of the material. The EELS performance at $300 \mathrm{kV}$ thus extends the usefulness of 


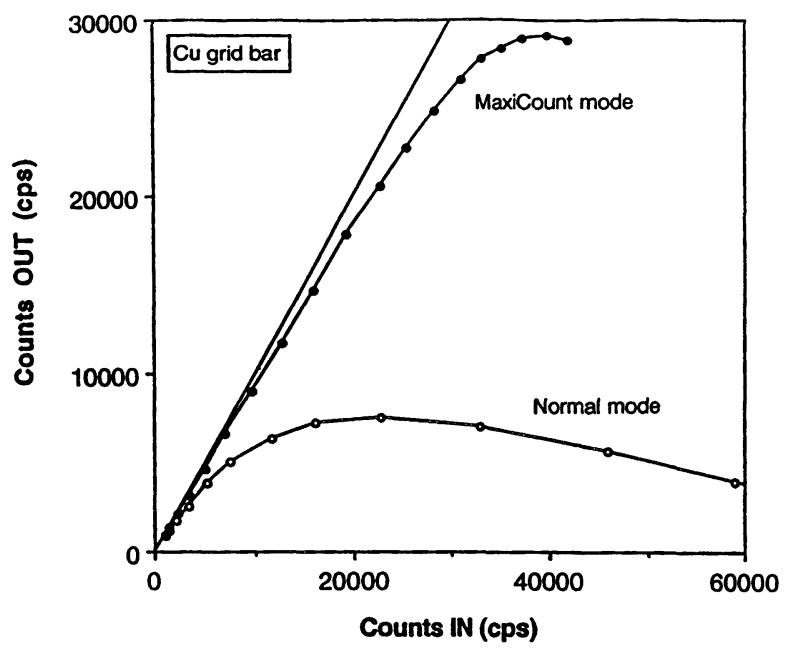

Fig. 6. - Input vs. output count rate with and without MaxiCount blanking (14 $\mu$ s processor time).

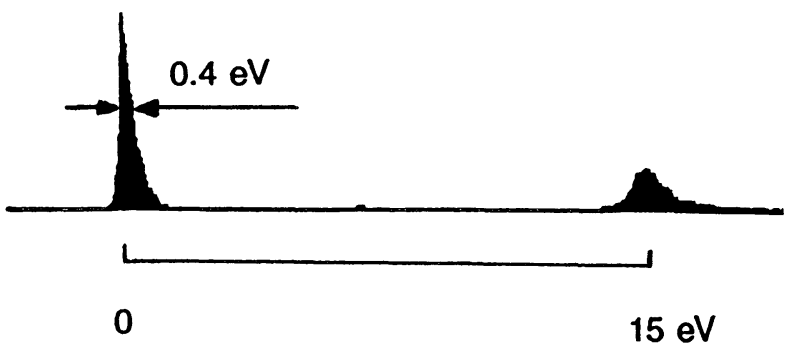

Fig. 7. - EELS spectrum of aluminium at $300 \mathrm{kV}$ : energy range $0-20 \mathrm{eV}$, zero-loss peak $0.4 \mathrm{eV} \mathrm{FWHM}$.

the technique to a much wider range of materials compared to $100 \mathrm{kV}$. From recent results at 100 $\mathrm{kV}$ [10] we can also expect $300 \mathrm{kV}$ EELS to achieve atomic resolution.

3.3 DifFRACTION PERFORMANCE. - The analytical HB603 has three projector lenses, including a high magnification final projector, to give a wide range of camera lengths at the YAG crystal screen. The screen is viewed by a lens-coupled CCD camera and/or a $35 \mathrm{~mm}$ film camera. An example of silicon convergent-beam diffraction patterns near the $<111>$ zone axis recorded at $300 \mathrm{kV}$ (Fig. 8) shows the fine structure is well resolved.

\section{High-resolution $Z$-contrast imaging.}

The high-angle annular dark-field (ADF) or Z-contrast technique is rapidly being established as an atomic-resolution analytical technique. Pennycook and co-workers [3] have shown that incoherent images of crystals at symmetrical orientations are directly interpretable at the atomic level and provide chemical information on individual columns of atoms in crystals and at interfaces.

As shown by Cowley [11], there is a fundamental advantage in resolution using incoherent (ADF) imaging compared to coherent (phase contrast) imaging. The resolution can be expressed 

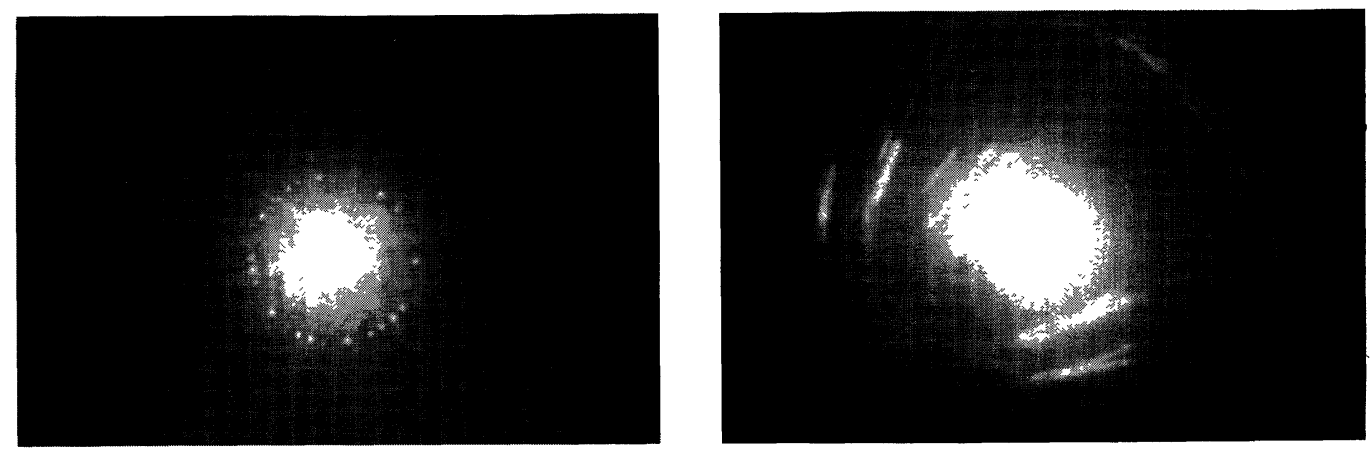

Fig. 8. - CBED patterns near $\mathrm{Si}<111>$ zone axis at low and high camera length, $300 \mathrm{kV}$.

by the equation:

$$
d=k\left(C_{\mathrm{s}} \lambda^{3}\right)^{1 / 4}
$$

where $k=0.66$ for coherent and $k=0.43$ for incoherent imaging conditions at Scherzer defocus ( $\lambda$ is the electron wavelength). Hence incoherent imaging gives an improvement in resolution of about $35 \%$. The corresponding contrast transfer functions (CTF) for the two imaging conditions (Fig. 9) clearly show very different characteristics: the coherent imaging CTF has the well-known contrast reversals beyond the Scherzer resolution limit, whereas the incoherent imaging function is monotonic and therefore directly interpretable beyond the Scherzer limit.

Coherent

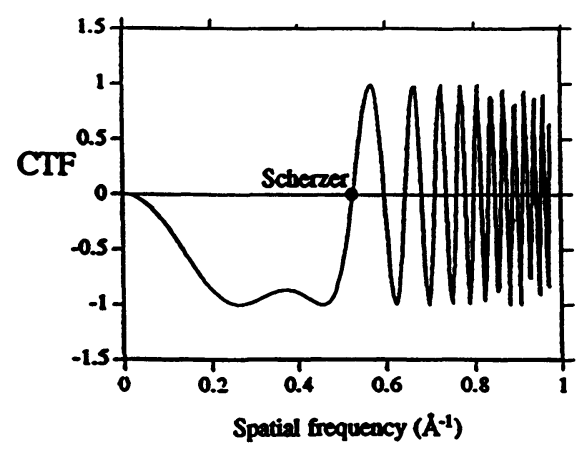

$$
\Delta \mathrm{f}=-506 \AA
$$

Incoherent

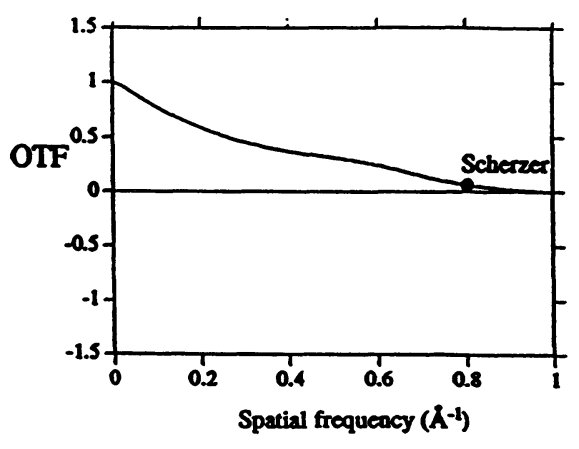

$\Delta f=-438 \AA \quad \alpha=9.36 \mathrm{mrad}$

Fig. 9. - Contrast transfer functions for coherent and incoherent imaging conditions at $300 \mathrm{kV}, C_{\mathrm{s}}=1.0$ mm.

In a STEM instrument the incoherent imaging conditions are easily satisfied by using an ADF detector of 25-50 mrad inner cut-off angle, depending on the material and acceleration voltage. At $300 \mathrm{kV}$ the incoherent image resolution is $0.125 \mathrm{~nm}$ for an objective lens of $1 \mathrm{~mm} C_{\mathrm{s}}$ value. 
The HB603U was designed to optimise the performance for ADF imaging. The detector arrangement (Fig. 10) is such that the annular cone of electrons is incident on a YAG screen and either the ADF signal is acquired by a photomultiplier or the diffraction pattern is viewed by a camera. The angular range in the ADF image - up to a maximum of $200 \mathrm{mrad}$ - can be adjusted by a projector lens and viewed by an intensified CCD camera.

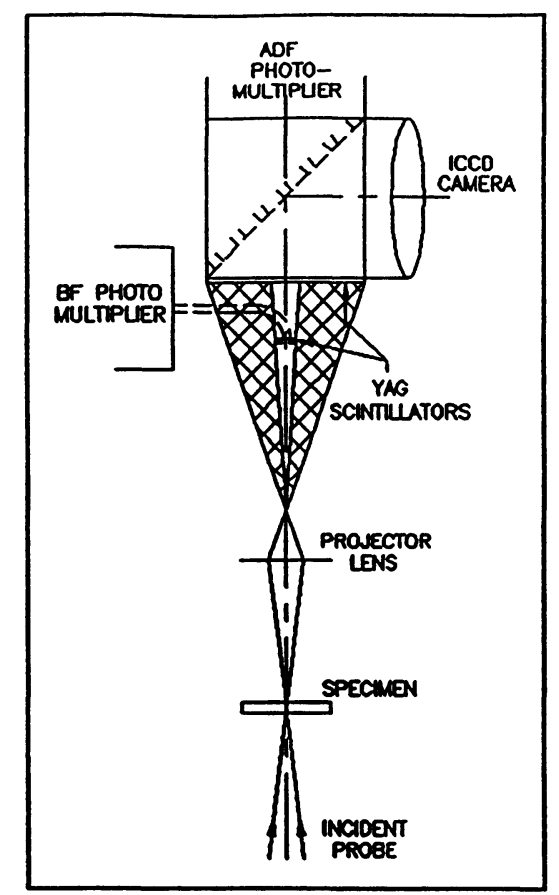

Fig. 10. - HB603U detector arrangement.

The instrument was designed for maximum stability of power supplies and mechanical components, particularly the stage. The detachable specimen holder design is well suited to meet the low drift requirements for imaging at magnifications of 20-50 million times. In practice, the doubletilt eucentric holder achieves a drift rate of less than $0.1 \mathrm{~nm}$ per minute. The objective lens was designed for a $1.0 \mathrm{~mm} C_{\mathrm{s}}$ value and $1 \mathrm{ppm}$ current stability; beam deflectors were designed to a stability corresponding to less than $0.01 \mathrm{~nm}$ deflection at the specimen.

As a resolution test of the instrument a single crystal specimen of silicon $<110>$ was used. Simulated images (Fig. 11) suggest that the dumbbells of $0.136 \mathrm{~nm}$ spacing should be resolved by the instrument. This was confirmed experimentally (Fig. 12) at the optimum defocus. Figure 12a shows the unprocessed image for a $20 \mathrm{sec}$ exposure and figure $12 \mathrm{~b}$ the same image after processing by the maximum entropy (MaxEnt) technique [12]. The fact that the Si dumbbells are clearly resolved shows that the $\mathrm{HB} 603 \mathrm{U}$ achieves a $Z$-contrast point resolution of $0.13 \mathrm{~nm}$. The processed image shows significant noise reduction and defines the atomic positions within $0.02 \mathrm{~nm}$. This suggests that there is information available at the 1 Angstrom level. 


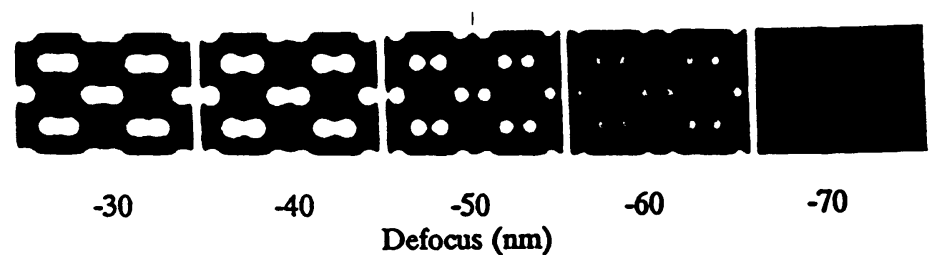

Fig. 11. - Simulated $\mathrm{Si}<110>\mathrm{ADF}$ images at -30 to $-70 \mathrm{~nm}$ defocus, $300 \mathrm{kV}$.

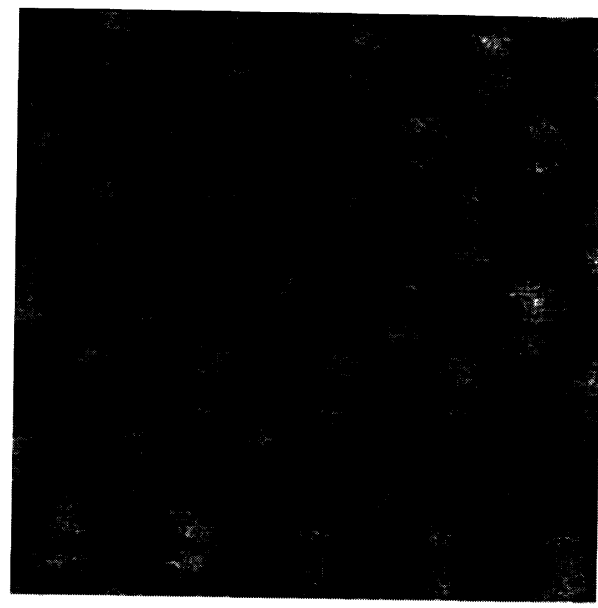

a

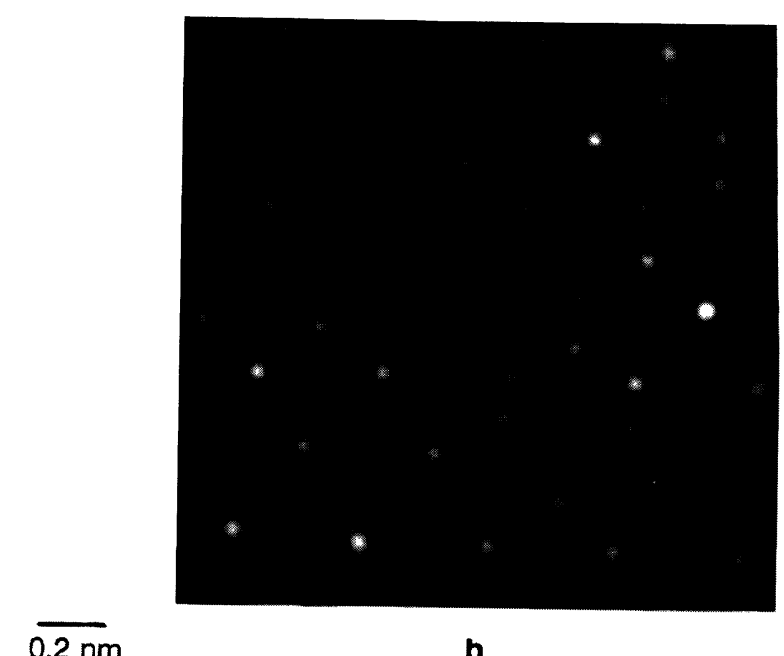

Fig. 12. - Experimental $\mathrm{Si}<110>$ image: a) raw data and b) MaxEnt processed image.

\section{Conclusions.}

With over 20 years of experience in field-emission STEM, $300 \mathrm{kV}$ instruments have been developed with ultimate analytical and high-resolution imaging performance. A number of factors have proved crucial for achieving this level of performance:

1) the cold field-emission gun which, with its narrow energy spread provides high energy resolution EELS,

2) the CFE gun and column which were designed for maximum probe brightness and minimum hole count in all analytical modes,

3) the mechanical and electronic stability of the column and low-drift stage design which guarantee high probe stability,

4) the UHV environment at the specimen which is essential for contamination-free analysis at sub-nanometre level.

5) the control system that is both flexible and user-friendly, and permits a wide range of users to achieve high quality, quantitative results. 


\section{Acknowledgements.}

The author would like to thank the VG Microscopes HB603 development team for their dedicated work in designing this instrument, and to Peter Bovey for comments on this manuscript.

\section{References}

[1] Titchmarsh J.M., Microbeam Analysis - 1988 (San Fran. Press, 1988) 65.

[2] Batson P.E., Microbeam Analysis - 1989 (San Fran. Press, 1989) 5.

[3] Pennycook S.J. and Jesson D.E., Ultramicroscopy 37 (1991) 14.

[4] Williams D.B. and Steel E.B., AEM-1987 (San Fran. Press, 1987) 228.

[5] Fiori C.E., Swyt C.R. and Ellis J.R., Microbeam Analysis - 1982, K.F.J. Heinrich Ed. (San Fran. Press, 1982) 57.

[6] Zaluzec N.J., Proc. 50th Ann. Meeting Electron Microscopy Society of America (San Fran. Press, 1992) 1466.

[7] Lyman C.E. and Ackland D.W., Proc.49th Ann. Meeting Electron Microscopy Society of America (San Fran. Press, 1991) 720.

[8] Nicholls A.W., Von Harrach H.S., Lyman C.E., Goldstein J.I., Williams D.B. and Ackland D.W., Inst. Phys. Conf. Ser. 138 (1993) 527.

[9] Egerton R.F., "Quantitative Electron Microscopy”, Proc. 25th Scottish Univ. Summer School in Physics, J.N. Chapman and A.J.Craven Eds. (SUSSP Publ., Edinburgh, 1984) 273.

[10] Browning N.D., Chisholm M.F. and Pennycook S.J., Nature 366 (1993) 143.

[11] Cowley J., Principles of Analytical Electron Microscopy, D.C. Joy, A.D.Romig and J.I. Goldstein Eds. (Plenum Press, New York, 1986) 77.

[12] Ferrige A.G., Seddon M.J., Pennycook S.J., Chisholm M.F. and Robinson D.R.T., Maximum Entropy and Bayesian Methods, Seattle 1991, C.R. Smith et al. Eds. (Kluwer Acad. Publ., 1992) 337. 